\title{
Designing Mass Immunization Clinics
}

\section{By Tanya Rac}

In an effort to protect the general population from novel influenza A H1N1 virus, health administrators and public health officials emphasized a well-known preventative measure - immunization. According to the World Health Organization, immunization is "undoubtedly one of the most cost-effective health achievements of modern times. It is one of the rare services that costs very little, but offers huge benefits for the health and well-being of populations"1. Unlike typical non-pandemic vaccines, the delivery of $\mathrm{H} 1 \mathrm{~N} 1$ vaccine required unique mass immunization plans ${ }^{2}$ Mass immunization clinics are the most effective method of providing immunization to a large number of people over a short period of time ${ }^{2}$. The planning and development of these clinics began long before the H1N1 pandemic had arrived. This report describes strategies that may be used to design pandemic immunization clinics.

Choosing a clinic location well suited to the needs of the public and the health officials is imperative for the success of the clinics. The team must consider whether the clinics are set up in rural or urban areas, the number of clinics to be set up, and whether the clinic locations will alternate over the immunization period. Examples of clinic sites are schools, leisure centers, church halls, malls, and universities. Some of these sites are already equipped with chairs, tables, Internet, and other objects/services required to set up a clinic site. Information technology is necessary for proper risk communication and record updates with various buildings, sites, cities, and with colleagues in other health jurisdictions.

A thorough transportation plan may be useful in preventing potential traffic dilemmas. For instance, the site should ensure adequate parking space for those driving personal vehicles. Public transportation plans should include looking into bus fare fees, re-routing of buses, and enhanced bus service.

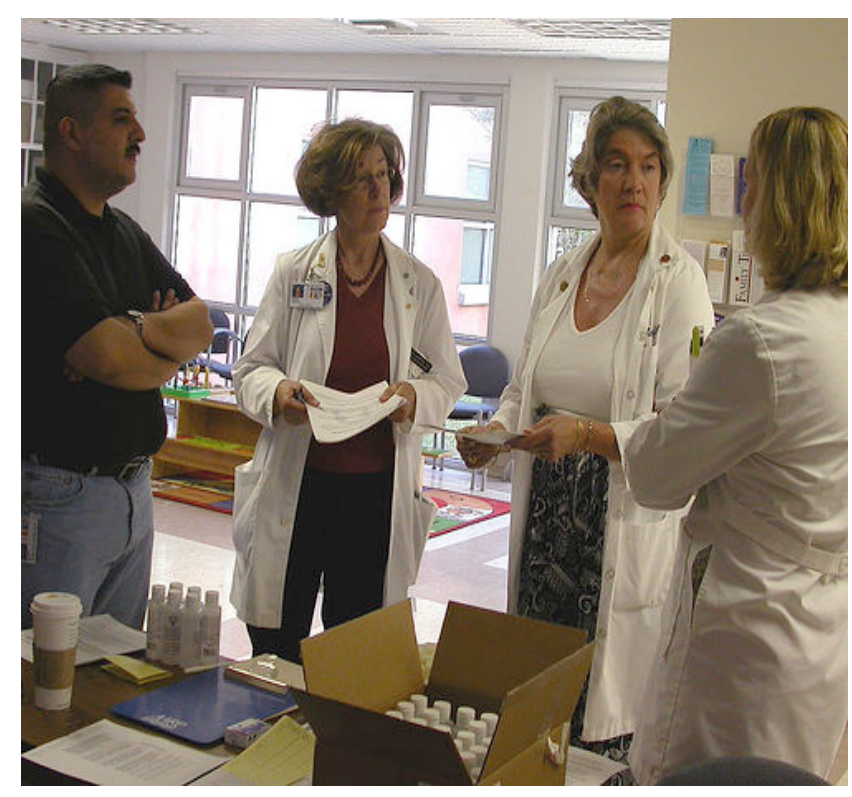

The vaccine cold chain is essential to maintain vaccine potency. It refers to the process used to maintain optimal conditions during the transport, storage, and handling of vaccines, starting at the manufacturer and ending with the administration of the vaccine to the client ${ }^{3}$. Vaccine should be stored between 2 to 8 degrees Celcius. Security of the vaccine during transportation, storage and distribution should include double-count vaccine sheets, locked storage facilities, and specific transport personnel.

During pandemics, there may be an overwhelming demand for certain immunization supplies. To ensure adequate stock, health regions may stockpile prior to the pandemic. Stockpiling may bring about its own complexities with storage space and designating staff responsible for keeping the inventory up-to-date and properly rotated. Ongoing use of immunization supplies generates large quantities of biomedical waste especially at mass immunization sites. Waste management sectors should have special protocols to carefully dispose this waste during the pandemic. Thorough 
cleaning in the clinics is important, as the "accumulation of dust, soil, and microbial contaminants on environmental surfaces is both aesthetically displeasing and a potential source of nosocomial infections" . Clinic custodians should be given biomedical housekeeping training manuals.

During the pandemic, the need for health services could exceed the available human resources $^{2}$. The disease impact and increased demand of health services can be projected by epidemiological analysis. Human resource should plan a schedule for health care professionals who will administer the vaccine based on the expected rates of vaccination, and provide adequate training courses.. Due to staff shortage and insufficient financial resources, the clinics may rely heavily upon volunteers. Volunteers should be given training sessions and orientation packages. This provides an ideal opportunity to involve a broad range of stakeholders especially from the community to maximize awareness. A strong communication plan among these participants is used to relay messages from the various levels of government and to share epidemiological findings.

Planning ahead in preparation for influenza pandemics, with its potentially high morbidity and mortality rates, is essential for hospital administrators and public health officials ${ }^{2}$. Adequate immunization decreases the incidence of the clinically ill, hospitalized, and the dead ${ }^{2}$. However, pandemics can present unexpected challenges. As a result, the mass immunization contingency plans must be flexible enough to adapt to the demands of the pandemic.

\section{Acknowledgements}

I would like to thank the Department of Population and Public Health Services of the Regina Qu'Appelle Health Region for their help in preparing this manuscript.

\section{References}

1. World Health Organization [Internet]. Vaccinepreventable diseases and immunization.

http://www.euro.who.int/vaccine/20081217_16) (accessed March 10, 2010).

2. Regina Qu'Appelle Health Region [RQHP]. (2009). RQHR pandemic pandemic preparedness plan version 2.0. Available from RGHR database. Regina, Saskatchewan: RQHR. (accessed July 3, 2009).
3. Public Health Agency of Canada [Internet]. National vaccine storage and handling guidelines for immunization providers. http://www.phac-

aspc.gc.ca/publicat/2007/nvshglp-ldemv/section1-eng.php (accessed May 27, 2009).

4. Tietjen L, Bossemeyer D, McIntosh N. Housekeeping. In: Infection prevention guidelines for healthcare facilities with limited resources. Chapter 16 - Housekeeping. JHPIEGO, an affiliate of Johns Hopkins University. Maryland; 2003.

http://www.reproline.jhu.edu/english/4morerh/4ip/IP_man ual/16_Housekeeping.pdf (accessed July 3, 2009).

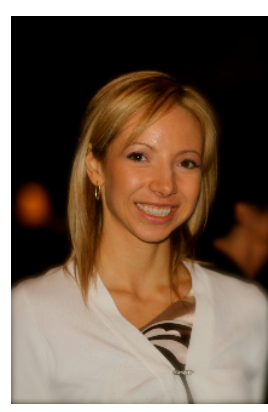

\section{Author Profile}

Tanya Rac received her MPH degree from the School of Public Health at the University of Saskatchewan, and is currently working for the Public Health Observatory, Saskatoon Health Region. She had also previously received a $\mathrm{BSc}$ degree from The University of Saskatchewan. 